\title{
Clinical relevance of procalcitonin and C-reactive protein as infection markers in renal impairment: a cross-sectional study
}

\author{
Ji Hyeon Park', Do Hee Kim', Hye Ryoun Jang ${ }^{1 *}$, Min-Ji Kim², Sin-Ho Jung², Jung Eun Lee', Wooseong Huh', \\ Yoon-Goo Kim', Dae Joong Kim and Ha Young $\mathrm{Oh}^{1}$
}

\begin{abstract}
Introduction: Although the clinical application of procalcitonin (PCT) as an infection marker in patients with impaired renal function (estimated glomerular filtration rate (eGFR) $<60 \mathrm{ml} / \mathrm{min} / 1.73 \mathrm{~m}^{2}$ ) has been increasing recently, it is unclear whether $\mathrm{PCT}$ is more accurate than C-reactive protein (CRP). We investigated the clinical value of CRP and PCT based on renal function.

Methods: From November 2008 to July 2011, a total of 493 patients who simultaneously underwent CRP and PCT tests were enrolled. The area under the receiver operating characteristic (ROC) curve and characteristics of both markers were analyzed according to infection severity and renal function.

Results: In patients with impaired renal function, the area under the ROC curve was 0.876 for CRP and 0.876 for PCT. In patients with infection, CRP levels differed depending on whether the infection was localized, septic, or severely septic, whereas PCT levels were higher in patients with severe sepsis or septic shock. In patients without infection, CRP did not correlate with eGFR, while PCT was negatively correlated with eGFR.

Conclusion: This study demonstrates that CRP is accurate for predicting infection in patients with impaired renal function. The study suggests that in spite of its higher cost, PCT is not superior to CRP as an infection marker in terms of diagnostic value.
\end{abstract}

\section{Introduction}

Severe infection and sepsis with accompanying dysfunction or failure of multiple organs are major causes of morbidity and mortality in patients with chronic kidney disease (CKD) $[1,2]$. These patients are particularly susceptible to infection because of functional deterioration of many components of the immune system [3]. However, it is difficult to differentiate between infectious causes and non-infectious causes of systemic inflammatory responses because of chronic elevation of inflammatory markers and nonspecific clinical symptoms that are common among patients with CKD $[4,5]$. In particular, the specificity of C-reactive protein (CRP) has been of concern because CRP has been found to be

* Correspondence: hyeryoun.jang@samsung.com

'Division of Nephrology, Department of Medicine, Samsung Medical Center Sungkyunkwan University School of Medicine, 81 Irwon-ro, Gangnam-gu, Seoul 135-710, Republic of Korea

Full list of author information is available at the end of the article nonspecifically elevated in chronic inflammatory conditions, such as atherosclerosis and CKD $[5,6]$.

Recently, procalcitonin (PCT) has become widely used for differentiating between infectious and non-infectious disease. Some studies have found that PCT is a more accurate marker for predicting infection than CRP in patients with impaired renal function as well as in those with normal renal function [7-9]. However, the diagnostic value or the best cutoff value of PCT in patients with impaired renal function or renal replacement therapy (RRT) remains unknown because the elimination route of PCT has not been described yet. Additionally, previous studies evaluating PCT in patients with impaired renal function included either an unrepresentative study sample or a very small sample size. Although a recent meta-analysis study reported that both CRP and PCT have acceptable specificity in diagnosing infection in patients with impaired renal function, that meta-analysis included studies with high heterogeneity in the study 
population [10], thus, there are still debates over the relative advantages of PCT compared to CRP, especially regarding diagnostic accuracy even with its higher cost $[10,11]$. The aim of this study is to evaluate the clinical relevance of PCT compared to CRP as an infection marker based on difference in renal function.

\section{Materials and methods Study population}

We performed a cross-sectional study to evaluate the diagnostic value of PCT compared to CRP in regard to renal function after obtaining approval from the Institutional Review Board (IRB) at Samsung Medical Center in compliance with the Declaration of Helsinki (IRB approval number: 2013-06-020). As we collected data retrospectively, patient consent was not applicable. Study participants were selected from the patient population at Samsung Medical Center, a 2,000-bed tertiary referral center in Seoul, Korea. Eligible participants were adults $(\geq 18$ years old) with suspected infection who underwent CRP and PCT tests simultaneously (within a 6-hour period) between 1 November 2008 and 31 July 2011. Patients were enrolled regardless of the hospitalization setting, such as ward, intensive care unit, or emergency room, to include patients with variable disease severity.

The total number of patients who received CRP and PCT tests within a 6-hour period upon admission during the study period was 25,075 . We recruited 23,819 of these eligible patients and then randomly selected 100 patients from each class of renal function based on estimated glomerular filtration rate (eGFR) to enroll patients evenly according to renal function (class 1: eGFR $\geq 90 \mathrm{~mL} / \mathrm{min} / 1.73 \mathrm{~m}^{2}$; class 2 : eGFR 60 to $90 \mathrm{~mL} / \mathrm{min} / 1.73 \mathrm{~m}^{2}$; class 3: eGFR 30 to $60 \mathrm{~mL} / \mathrm{min} / 1.73 \mathrm{~m}^{2}$, class 4 : eGFR 15 to $30 \mathrm{~mL} / \mathrm{min} / 1.73$ $\mathrm{m}^{2}$; class 5 : eGFR $\leq 15 \mathrm{~mL} / \mathrm{min} / 1.73 \mathrm{~m}^{2}$ without RRT; class 6: eGFR $\leq 15 \mathrm{~mL} / \mathrm{min} / 1.73 \mathrm{~m}^{2}$ with hemodialysis (HD); or class 7: eGFR $\leq 15 \mathrm{~mL} / \mathrm{min} / 1.73 \mathrm{~m}^{2}$ with peritoneal dialysis (PD)). After excluding patients with an unclear clinical course of infection, a total of 493 patients were ultimately enrolled in this study.

The randomly selected patients were divided into two groups based on their infection status as determined by a review of their medical records. Patients were defined as having an infection when a clinically definable source of infection was present, as confirmed by microbiology tests and/or positive blood cultures. In cases of suspected PD peritonitis, a diagnosis was made if the peritosol white blood cell count was greater than 100 cells/ $\mathrm{mm}^{3}$ and the percentage of neutrophils was greater than $50 \%$, even if no microbe was identified [12]. Cases with clinical ambiguities or obscurities that made it difficult to classify infection status were excluded. Thus, cases with no microbiologically-confirmed culture results to accompany a clinical diagnosis of infection were excluded. Likewise, cases with microbiologically-confirmed culture results but no clinical diagnosis of infection were also excluded. Additionally, cases without a conclusive clinical diagnosis of systemic inflammatory response or organ failure were excluded from both groups.

Important clinical and laboratory variables, such as age, sex, preexisting underlying disease, clinical diagnosis, severity of infectious condition, microbiology of culture from infection source, hematologic data, and chemistry data, were collected when CRP and PCT tests were conducted. The American College of Chest Physicians/Society of Critical Care Medicine Consensus Conference definition of sepsis stage was used to classify the severity of infection as sepsis, severe sepsis, or septic shock [13].

The primary outcome was a comparison between the reliability of CRP and PCT as diagnostic markers of infection in patients with impaired renal function. The secondary outcomes included the best cutoff value of CRP and PCT levels in patients with impaired renal function, the association between CRP and PCT levels and infection severity in patients with impaired renal function, and the association between CRP and PCT levels and renal function in the absence of infection.

\section{Measurements}

Renal function was assessed based on eGFR levels using the modification of diet in renal disease (MDRD) equation. Serum CRP concentrations were measured with an immunoturbidimetric assay (CRPL3, Roche Diagnostics, Indianapolis, IN, USA) and the lower reference limit was $0.3 \mathrm{mg} / \mathrm{dL}$. Serum PCT concentrations were measured with an enzyme-linked fluorescent assay (Brahms Diagnostica $\mathrm{GmbH}$, Berlin, Germany) and the lower reference limit was $0.05 \mathrm{ng} / \mathrm{mL}$.

\section{Statistical analyses}

For statistical analyses, SPSS PAWS version 20.0 (SPSS Inc., Chicago, IL, USA) was used. For continuous variables, data are shown as the median and IQR if the data were not normally distributed. Categorical data are shown as the number and percentage. Continuous variables were analyzed using the Mann-Whitney $U$-test. The KruskalWallis test and Tukey test using ranks for post-hoc comparison were used for multiple comparisons. Categorical data were analyzed using the chi-square test. To compare the predictive ability of CRP and PCT for infection, receiver operating characteristic (ROC) curves and the areas under the respective curve (AUC) were calculated. AUCs were compared using the nonparametric approach of DeLong et al. for two correlated AUCs [14]. Spearman correlation analysis was used for nonparametric data. $P$-values less than 0.05 were considered statistically significant. 


\section{Results}

Among the 700 patients assessed, 207 patients were excluded because of clinical ambiguities about their infection status, as described in Materials and methods. A total of 493 patients were finally analyzed. Patients were divided into two groups based on renal function (group I: eGFR $\geq 60 \mathrm{~mL} / \mathrm{min} / 1.73 \mathrm{~m}^{2}$; group II: eGFR $<60 \mathrm{~mL} /$ $\left.\min / 1.73 \mathrm{~m}^{2}\right)$. In group I, 73 patients $(48 \%)$ had an infection and 78 patients (52\%) did not have an infection, and in group II, 186 patients (46\%) had an infection and 156 patients (54\%) did not have an infection.

Baseline characteristics are summarized in Table 1. There were no significant differences in age, sex, or major comorbidities (such as diabetes mellitus (DM) or hypertension) and renal function based on infection status (Table 1). Disease categories are shown in Table 2. The most common infectious disease in the sample population was urinary tract infection $(26.6 \%)$, followed by intra-abdominal infection (17.0\%) (Table 2). The most common non-infectious disease was cardiovascular disease (26.1\%) (Table 2).

The median values of CRP and PCT are shown in Table 3. The median values of CRP and PCT were significantly higher in patients with infectious diseases (CRP: $P<0.001$; PCT: $P<0.001)$. Sub-analyses of CRP and PCT based on renal function showed that both markers were significantly elevated in patients with infection (Table 3).

The diagnostic value of CRP and PCT for predicting infection was compared using the ROC curve. The AUC for the diagnosis of infection versus non-infection was 0.819 (95\% CI 0.782, 0.856) for CRP and 0.831 (95\% CI $0.795,0.866$ ) for PCT (Figure 1a). For group I, the AUC was 0.684 (95\% CI $0.587,0.782)$ for CRP and 0.766 (95\% CI $0.681,0.851$ ) for PCT (Figure 1b). For group II, the AUC was 0.876 (95\% CI 0.839, 0.912) for CRP and 0.876 (95\% CI 0.839, 0.912) for PCT (Figure 1c). There were no differences between the AUCs of CRP and PCT in the two groups (total: $P=0.59$ for all patients; $P=0.12$ for group I; $P=0.9$ for group II). When the AUC of CRP and PCT was analyzed further in patients with systemic inflammatory response syndrome (SIRS) (excluding patients without SIRS from both groups), CRP still showed no inferiority to PCT in differentiating infection. In patients with SIRS, the AUC of CRP was 0.804 (95\% CI $0.759,0.850)$ and that of PCT was 0.802 (95\% CI 0.757, 0.847). In SIRS patients with normal renal function, the AUC of CRP was 0.629 (95\% CI 0.532, 0.725) and that of PCT was 0.694 (95\% CI 0.603, 0.785). In SIRS patients with impaired renal function, the AUC of CRP was 0.890 (95\% CI $0.849,0.931)$ and that of PCT was 0.858 (95\% CI 0.811, 0.906). CRP was not inferior to PCT in patients with SIRS.

Additionally, to evaluate whether the diagnostic accuracy could be increased using CRP and PCT together, the AUC of both CRP and PCT together was compared with the AUC of CRP alone. The AUC of CRP and PCT measurements together was significantly higher than the AUC of CRP alone (AUC of both CRP and PCT versus AUC of CRP alone: 0.858 versus 0.819 in all patients, $P<0.001$; 0.758 versus 0.684 for group I, $P=0.01 ; 0.899$ versus 876 for group II, $P=0.01$ ).

The best cutoff value for diagnosing infection in patients with impaired renal function (group II) was $3.08 \mathrm{mg} / \mathrm{dL}$ for CRP and $1.1 \mathrm{ng} / \mathrm{mL}$ for PCT. In patients with normal renal function, the best cutoff value was $2.49 \mathrm{mg} / \mathrm{dL}$ for CRP and $0.08 \mathrm{ng} / \mathrm{mL}$ for PCT.

The association between CRP and PCT levels and the severity of infection in patients with impaired renal function (group II) was also analyzed. Group II patients with infectious diseases were categorized depending on the severity of their infections defined as no SIRS, sepsis, severe sepsis, or septic shock. Both CRP and PCT showed significant correlation with infection severity using Spearman correlation (CRP: $P<0.001, r=0.378$; PCT: $P<0.001, r=0.414)$. The Kruskal-Wallis test was also performed to analyze the differences in CRP and PCT among four subgroups. There were differences in CRP and PCT among four subgroups $(P<0.001$ for both CRP and PCT) (Figure 2). However, the Tukey test using ranks for post-hoc comparison showed no

Table 1 Baseline characteristics

\begin{tabular}{|c|c|c|c|c|c|c|c|c|c|}
\hline & \multicolumn{3}{|l|}{ Total } & \multicolumn{3}{|l|}{ Group I } & \multicolumn{3}{|l|}{ Group II } \\
\hline & Infection- & Infection+ & $P$ & Infection- & Infection+ & $P$ & Infection- & Infection+ & $P$ \\
\hline Number & 234 & 259 & - & 78 & 73 & - & 156 & 186 & - \\
\hline Age, yr & $61(24)$ & $62(20)$ & 0.84 & $52(22)$ & $51(32)$ & 0.9 & $69(24)$ & $66(18)$ & 0.49 \\
\hline Female, \% & $90(38.5)$ & $115(44.4)$ & 0.20 & $30(38.5)$ & $33(45.2)$ & 0.83 & $60(38.5)$ & $82(44.1)$ & 0.64 \\
\hline $\mathrm{DM}, \%$ & $68(29.1)$ & $85(32.8)$ & 0.38 & $7(9.0)$ & $16(21.9)$ & 0.08 & $61(39.1)$ & $69(37.1)$ & 0.9 \\
\hline HTN, \% & $104(44.4)$ & $117(45.2)$ & 0.9 & $15(19.2)$ & $19(26.0)$ & 0.67 & $89(57.1)$ & $98(52.7)$ & 0.89 \\
\hline eGFR, mL/min/1.73 $\mathrm{m}^{2}$ & $29.9(68.8)$ & $22.2(63.0)$ & 0.13 & $104.6(59.6)$ & $100.2(38.1)$ & 0.9 & $14.4(20.3)$ & $14.4(25.3)$ & 0.78 \\
\hline
\end{tabular}

Categorical data were analyzed using the chi-square test and continuous variables were analyzed using the Mann-Whitney $U$-test. Data represent median (IQR) or number (percentage). Group I: eGFR $\geq 60 \mathrm{~mL} / \mathrm{min} / 1.73 \mathrm{~m}^{2}$, Group II: eGFR $<60 \mathrm{~mL} / \mathrm{min} / 1.73 \mathrm{~m}^{2}$. DM, diabetes mellitus; HTN, hypertension; eGFR, estimated glomerular filtration rate. 
Table 2 Disease categories

\begin{tabular}{|c|c|}
\hline Disease category & Number (\%) \\
\hline \multicolumn{2}{|l|}{ Disease categories in patients with infection } \\
\hline Urinary tract infection & $69(26.6)$ \\
\hline Intra-abdominal infection & $44(17.0)$ \\
\hline Pneumonia & $39(15.1)$ \\
\hline Peritonitis & $29(11.2)$ \\
\hline Bacteremia with unknown focus & $27(10.4)$ \\
\hline Skin and soft tissue infection & $26(10.0)$ \\
\hline Vascular infection & $13(5.0)$ \\
\hline Catheter related infection & $8(3.1)$ \\
\hline Others & $4(1.5)$ \\
\hline Total & $259(100)$ \\
\hline \multicolumn{2}{|l|}{ Disease categories in patients without infection } \\
\hline $\begin{array}{l}\text { Cardiovascular disease (atrial fibrillation, myocardial } \\
\text { infarction, heart failure) }\end{array}$ & $61(26.1)$ \\
\hline $\begin{array}{l}\text { Renal disease (rhabdomyolysis, thrombotic } \\
\text { thrombocytopenic purpura-hemolytic uremic syndrome) }\end{array}$ & $35(15.0)$ \\
\hline $\begin{array}{l}\text { Gastroenterohepatic disease (gastrointestinal bleeding, } \\
\text { toxic hepatitis) }\end{array}$ & $34(14.5)$ \\
\hline $\begin{array}{l}\text { Hemato-oncologic disease (lymphoma B symptom, } \\
\text { sarcoidosis) }\end{array}$ & $30(12.8)$ \\
\hline $\begin{array}{l}\text { Respiratory disease (chronic obstructive pulmonary } \\
\text { disease, interstitial lung disease) }\end{array}$ & $19(8.1)$ \\
\hline Rheumatologic disease (gout, adult onset Still's disease) & $15(6.4)$ \\
\hline $\begin{array}{l}\text { Neuropsychiatric disease (cerebral infarction, intracranial } \\
\text { hemorrhage) }\end{array}$ & $13(5.6)$ \\
\hline $\begin{array}{l}\text { Endocrinological disease (adrenal insufficiency, } \\
\text { hypoglycemia, diabetic ketoacidosis) }\end{array}$ & $8(3.4)$ \\
\hline Musculoskeletal disease (fractures) & $4(1.7)$ \\
\hline Others (drug fever, pain shock) & $15(6.4)$ \\
\hline Total & $234(100)$ \\
\hline
\end{tabular}

significant differences in CRP between severe sepsis and septic shock $(P=0.549)$ (Figure 2a). In PCT, the Tukey test using ranks for post-hoc comparison showed no significant differences between absence of SIRS and sepsis $(P=0.851)$ (Figure 2b).

The correlation between CRP, PCT, and renal function was analyzed in patients without infection. Patients receiving renal replacement therapy were excluded in this analysis. CRP was not correlated with eGFR $(P=0.70)$, whereas PCT was inversely correlated with eGFR $(r=$ $0.247, P=0.01$ ) (Figure 3).

\section{Discussion}

This study demonstrated that PCT is not superior to CRP for diagnostic accuracy, nor is it more reliably associated with infection severity considering renal function. CRP had diagnostic accuracy similar to PCT in patients with impaired renal function as well as in those with normal renal function. In patients with infection, CRP levels were higher in cases with less severe infection, which was a significantly different result than the levels observed among patients without SIRS, with sepsis, and with severe sepsis. PCT increased with more severe infection and there were no significant differences in PCT levels between patients with and without SIRS. Among patients without infection, CRP was not correlated with eGFR, while PCT was inversely correlated with eGFR.

A recent meta-analysis found that PCT was not superior to CRP in patients with impaired renal function [10]. In that study, hierarchical summary ROC curves had an AUC of 0.88 (95\% CI 0.83, 0.92) for CRP and 0.89 (95\% CI $0.86,0.92)$ for PCT, which were similar to our results (AUC: $0.876,95 \%$ CI $0.839,0.912$ for CRP; AUC: 0.876, 95\% CI $0.839,0.912$ for PCT). Also in a recent study including a relatively smaller homogenous population of CKD patients, AUC was 0.860 (95\% CI 0.712, 1.000) for CRP and 0.911 (95\% CI 0.773, 1.000) for PCT. There was also no significant difference in $\operatorname{AUC}(P=0.53, z$-statistic $=$ $0.62,95 \%$ CI $0.110,0.211$ ) [15].

In addition to the finding that $\mathrm{PCT}$ is not superior to CRP, it is also more costly: PCT testing is eight times more expensive than CRP testing in Korea, and two to four times more expensive in the US and Europe. Among patients with normal renal function, our data showed higher sensitivity and specificity of PCT compared to CRP, which is consistent with the findings of previous studies [8,9]. Although the kinetics of PCT, including elimination route and mechanism, have not been fully elucidated, it is possible that impaired renal function or dialysis may influence PCT because of its low molecular weight (13 kDa) [16]. Previous studies that evaluated the influence of renal function or dialysis on PCT yielded inconsistent results [7,17-22]. Our study demonstrated a weakly significant correlation between PCT and eGFR in non-infected patients. This result implies that PCT may be also influenced by renal function. Furthermore, PCT was not distinctively elevated in patients with less severe infections, and the basal level of PCT increased in patients with impaired renal function $[7,8,17-19,22]$; thus, a higher cutoff value for PCT was suggested for use in patients with impaired renal function $[7,10,18]$. Similarly, in our study, the best cutoff values of PCT for diagnosing infection were $1.1 \mathrm{ng} /$ $\mathrm{mL}$ for patients with impaired renal function and $0.08 \mathrm{ng} /$ $\mathrm{mL}$ for patients with normal renal function.

Previous studies that investigated the association between infection severity and CRP or PCT did not specifically explore these relationships in patients with impaired renal function exclusively $[8,23,24]$. Still, those studies also found that CRP levels were higher in patients with mild organ dysfunction and sepsis, but CRP levels did not increase significantly with progression toward more severe 
Table 3 C-reactive protein (CRP) and procalcitonin (PCT) levels by renal function and infection status

\begin{tabular}{|c|c|c|c|c|c|c|}
\hline & \multicolumn{3}{|l|}{$\mathrm{CRP}, \mathrm{mg} / \mathrm{dL}$} & \multicolumn{3}{|l|}{$\mathrm{PCT}, \mathrm{ng} / \mathrm{mL}$} \\
\hline & Infection- & Infection+ & $P$ & Infection- & Infection+ & $P$ \\
\hline Total & $1.12(3.63)$ & $10.62(17.94)$ & $<0.001$ & $0.15(0.42)$ & $2.86(22.73)$ & $<0.001$ \\
\hline Group I (eGFR $\geq 60$ ) & $2.38(8.46)$ & $8.42(10.82)$ & $<0.001$ & $0.05(0.13)$ & $0.45(4.21)$ & $<0.001$ \\
\hline Group II (eGFR <60) & $0.83(2.48)$ & $12.08(20.02)$ & $<0.001$ & $0.25(0.53)$ & $4.76(32.37)$ & $<0.001$ \\
\hline $\mathrm{eGFR} \geq 90$ & $2.38(5.36)$ & $6.09(10.36)$ & 0.001 & $0.05(0.10)$ & $0.21(1.98)$ & $<0.001$ \\
\hline $60 \leq$ eGFR $<90$ & $2.55(11.45)$ & $11.06(16.52)$ & 0.03 & $0.07(0.20)$ & $1.28(20.37)$ & $<0.001$ \\
\hline $30 \leq \mathrm{eGFR}<60$ & $0.96(2.37)$ & $12.19(16.46)$ & $<0.001$ & $0.09(0.22)$ & $2.88(19.80)$ & $<0.001$ \\
\hline $15 \leq \mathrm{eGFR}<30$ & $0.75(2.28)$ & $19.86(21.69)$ & $<0.001$ & $0.17(0.42)$ & $23.22(77.22)$ & $<0.001$ \\
\hline \multicolumn{7}{|l|}{ eGFR $<15$} \\
\hline Without RRT & $0.62(2.65)$ & $18.82(16.54)$ & $<0.001$ & $0.25(0.53)$ & $14.44(65.76)$ & $<0.001$ \\
\hline $\mathrm{HD}$ & $1.89(3.53)$ & $10.19(22.12)$ & $<0.001$ & $0.55(0.80)$ & $7.37(36.34)$ & $<0.001$ \\
\hline PD & $0.40(1.33)$ & $3.30(9.78)$ & $<0.001$ & $0.40(0.45)$ & $1.31(10.74)$ & 0.001 \\
\hline
\end{tabular}

Statistics were analyzed by the Mann Whitney U-test. Data represent median (IQR). Group I: eGFR $\geq 60 \mathrm{~mL} / \mathrm{min} / 1.73 \mathrm{~m}{ }^{2}, \mathrm{Group} \mathrm{II:} \mathrm{eGFR} \mathrm{<60} \mathrm{mL} / \mathrm{min} / 1.73 \mathrm{~m}{ }^{2}$. eGFR, estimated glomerular filtration rate; RRT, renal replacement therapy; HD, hemodialysis; PD, peritoneal dialysis.

stages of disease [8]. In contrast, PCT was reported to be modestly higher in cases of local infection and cases of infection without multiple organ failure [25]. Similarly, in our study, CRP levels were significantly different between impaired renal function cases without SIRS, with sepsis, and with severe sepsis. However, there was no significant difference between CRP levels in cases of severe sepsis and septic shock. In contrast with CRP results, PCT levels increased in patients with more severe organ dysfunction, severe sepsis, or septic shock. These differences are possibly due to different sources of CRP and PCT during the inflammatory process. CRP is produced only by de novo hepatic synthesis [6]. Therefore, CRP levels might not be increased further in patients with hepatic dysfunction, even under conditions of severe infection, such as septic shock or severe sepsis. Leukocytes are thought to be the source of PCT during sepsis, although there is still controversy regarding the origins of PCT [26]. During less severe infection, no SIRS, or sepsis, we observed a subtle increase in PCT levels, probably due to less specific stimuli to the leukocytes. These different responses, depending on the severity of infection, suggest that CRP would be a more valuable infection marker because CRP increases gradually, even in less severe infection, allowing for differential diagnosis of SIRS or no SIRS.

Interestingly, our study showed the best accuracy of CRP and PCT in patients with renal impairment. We compared the proportion of patients with septic shock with other subgroups (no SIRS, sepsis, and severe sepsis) using the chi-square test. The proportion of septic shock was greater in patients with renal impairment than those with normal renal function (the proportion of septic shock: $30 \%$ in renal impairment, $11 \%$ in normal renal function, $P=0.001$ ). However, there was no difference in the overall severity of infection between renal impairment and normal renal function groups when analyzed by the Wilcoxon rank sum test $(P=0.133)$. Although the best accuracy of CRP and PCT in patients with renal impairment might be affected by the partial proportional difference of infection severity, we believe that this point did not substantially compromise the aim of this study to compare CRP and PCT as an infection marker.

This study has several limitations. First, there may have been some selection bias because of the retrospective design. Because there are no gold standard criteria for diagnosing infection, there might be some misclassifications of infection status in this study. However, the definition of infection has an inevitable methodological limitation in all similar studies. In order to overcome this limitation, all ambiguous cases were excluded from our study. Second, unexpected factors that could affect CRP and PCT levels were not concurrently analyzed in this study. However, cardiovascular disorder, a common factor known to increase CRP and PCT, was thoroughly analyzed alongside renal function, and no significant association was found between cardiovascular disease and eGFR $(P=0.64)$. Third, our study sample was not large. Nonetheless, we enrolled a comparable number of patients across each stage of renal function, including dialysis patients. Furthermore, to our knowledge, this study included the largest study population among single-center studies evaluating the diagnostic value of PCT in patients with impaired renal function, although the patient population of our study might be heterogeneous because of various hospitalization settings including wards, intensive care units, or 

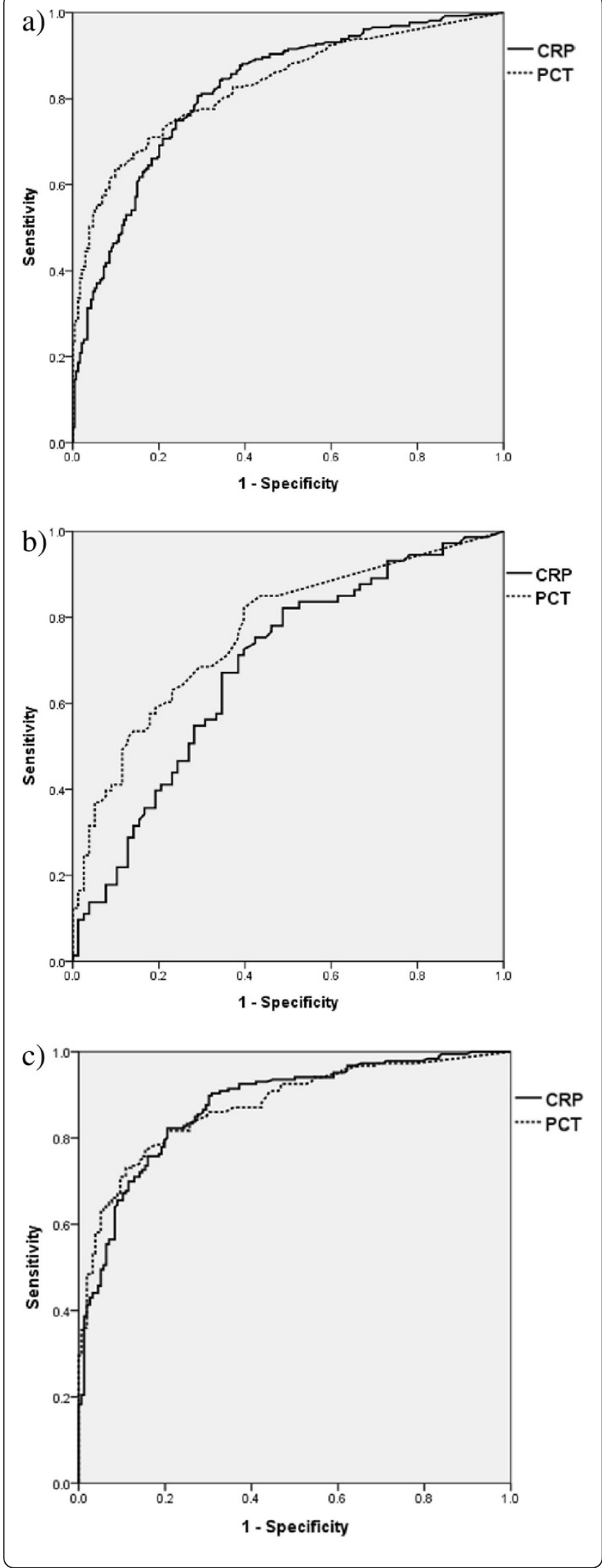

Figure 1 Receiver operating characteristic curves of C-reactive protein (CRP) and procalcitonin (PCT) for predicting infection. CRP is shown as solid lines, PCT as broken lines. (a) Receiver operating characteristic (ROC) curves for predicting infection in all patients. CRP: area under the curve (AUC) 0.819 ( $95 \% \mathrm{Cl} 0.782$, 0.856 ), best cutoff value $3.08 \mathrm{mg} / \mathrm{dL}$; sensitivity $81 \%$, specificity 71\%; positive predictive value (PPV) 0.75 , negative predictive value (NPV) 0.77. PCT: AUC, and best cutoff value 0.831 (95\% Cl 0.795, 0.866 ) and $1.1 \mathrm{ng} / \mathrm{mL}$, respectively; sensitivity and specificity $64 \%$ and $90 \%$, respectively; PPV and NPV 0.88 and 0.69 , respectively. (b) ROC curves for prediction of infection in patients with normal renal function (group I). CRP: AUC, and best cutoff value $0.684(95 \% \mathrm{Cl}$ $0.587,0.782)$ and $2.49 \mathrm{mg} / \mathrm{dL}$, respectively; sensitivity and specificity $82 \%$ and $51 \%$, respectively; PPV and NPV 0.61 and 0.75 , respectively. PCT: AUC, and best cutoff value $0.766(95 \% \mathrm{Cl} 0.681,0.851)$ and 0.08 $\mathrm{ng} / \mathrm{mL}$, respectively; sensitivity and specificity $82 \%$ and $60 \%$, respectively; PPV and NPV 0.66 and 0.78 , respectively. (c) ROC curves for prediction of infection in patients with impaired renal function. CRP: AUC, and best cutoff value 0.876 ( $95 \% \mathrm{Cl} 0.839,0.912)$ and $3.08 \mathrm{mg} / \mathrm{dL}$, respectively; sensitivity and specificity $82 \%$ and 79\%, respectively; PPV and NPV 0.83 and 0.79 , respectively. PCT: AUC, and best cutoff value $0.876(95 \% \mathrm{Cl} 0.839,0.912)$ and $1.1 \mathrm{ng} /$ $\mathrm{mL}$, respectively; sensitivity and specificity $73 \%$ and $89 \%$, respectively; PPV and NPV 0.89 and 0.74 , respectively.

emergency rooms. Nonetheless, as a single-center study, the measurement techniques and diagnostic criteria for infection used in our study were consistent across all participants.

\section{Conclusions}

In conclusion, this study suggests that in spite of its higher cost, PCT is not superior to CRP as an infection marker in terms of diagnostic value. The sensitivity and specificity of PCT and CRP were comparable in our analyses; however, PCT levels had an inverse relationship with eGFR in patients with renal insufficiency but no infection. Finally, considering that PCT levels were not significantly different between patients without SIRS and patients with sepsis, CRP would be a more valuable marker of infection in those patients.

\section{Key messages}

- Both CRP and PCT are useful to distinguish infectious conditions from non-infectious conditions, not only in patients with normal renal function, but also in patients with impaired renal function.

- Although the accuracy of CRP and PCT as infection markers was comparable in patients with impaired renal function, there was an inverse relationship between PCT and eGFR in patients without infection.

- PCT is not superior to CRP as an infection marker in terms of diagnostic value in patients with impaired renal function despite its higher cost. 

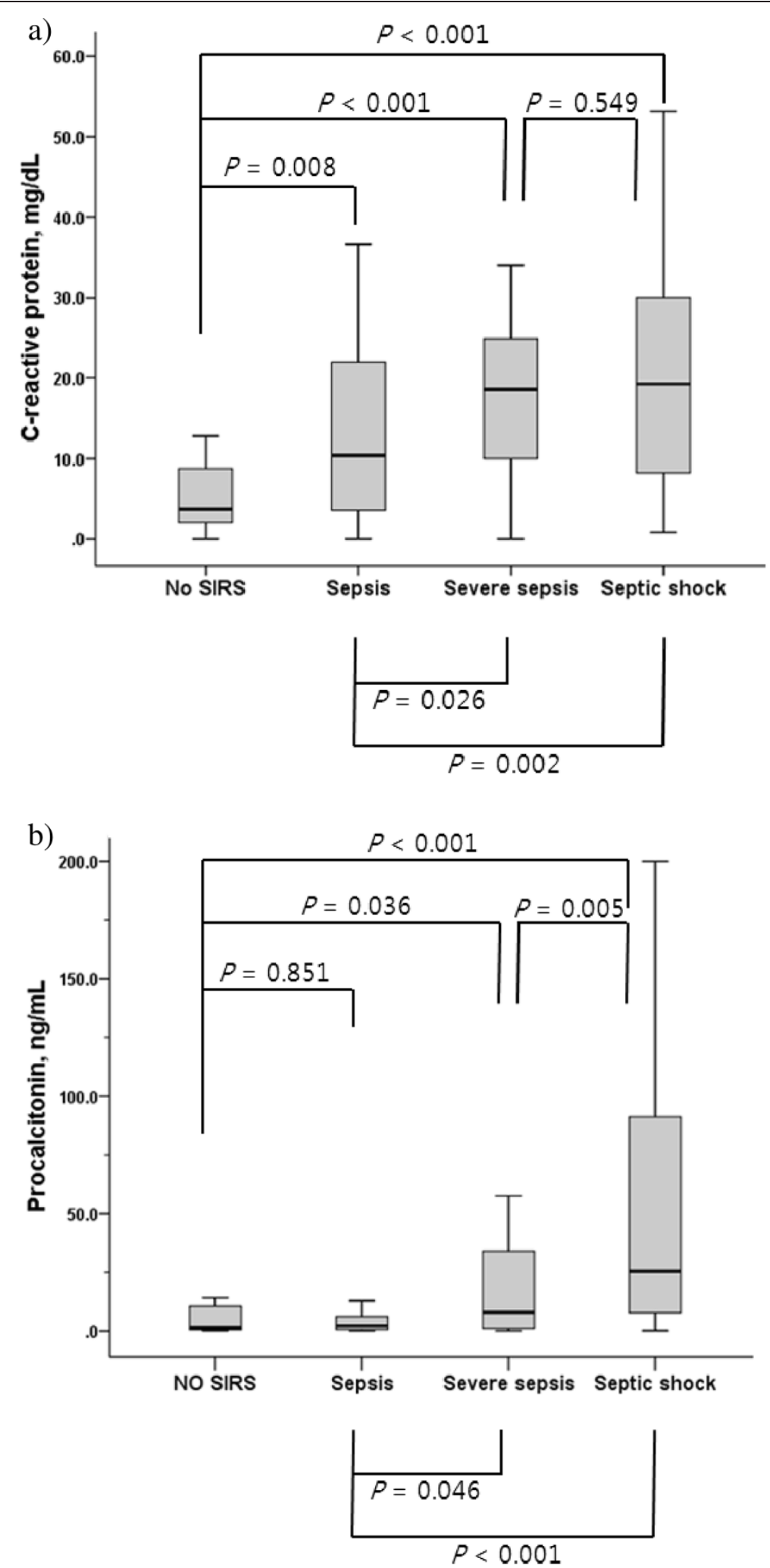

Figure $\mathbf{2}$ (See legend on next page.) 
(See figure on previous page.)

Figure 2 The association between C-reactive protein (CRP) and procalcitonin (PCT) levels and the severity of infection in patients with impaired renal function. The differences in CRP and PCT among four subgroups were analyzed using the Kruskal-Wallis test. (a) CRP increased depending on the infection severity $(P<0.001)$. However, there was no difference in CRP between severe sepsis and septic shock $(P=0.549)$. The median (IQR) CRP in each subgroup was as follows: 3.74 (7.27) when there was no systemic inflammatory response syndrome (SIRS), 10.41 (18.82) in sepsis, 18.59 (15.43) in severe sepsis, and 19.26 (21.94) in septic shock. (b) PCT increased depending on the infection severity $(P<0.001)$. However, PCT was not significantly higher in patients with sepsis than in those without SIRS $(P=0.851)$. The median (IQR) PCT in each subgroup was as follows: 1.52 (10.78) in the absence of SIRS, 2.22 (5.51) in sepsis, 7.96 (34.97) in severe sepsis, and 25.41 (85.11) in septic shock.
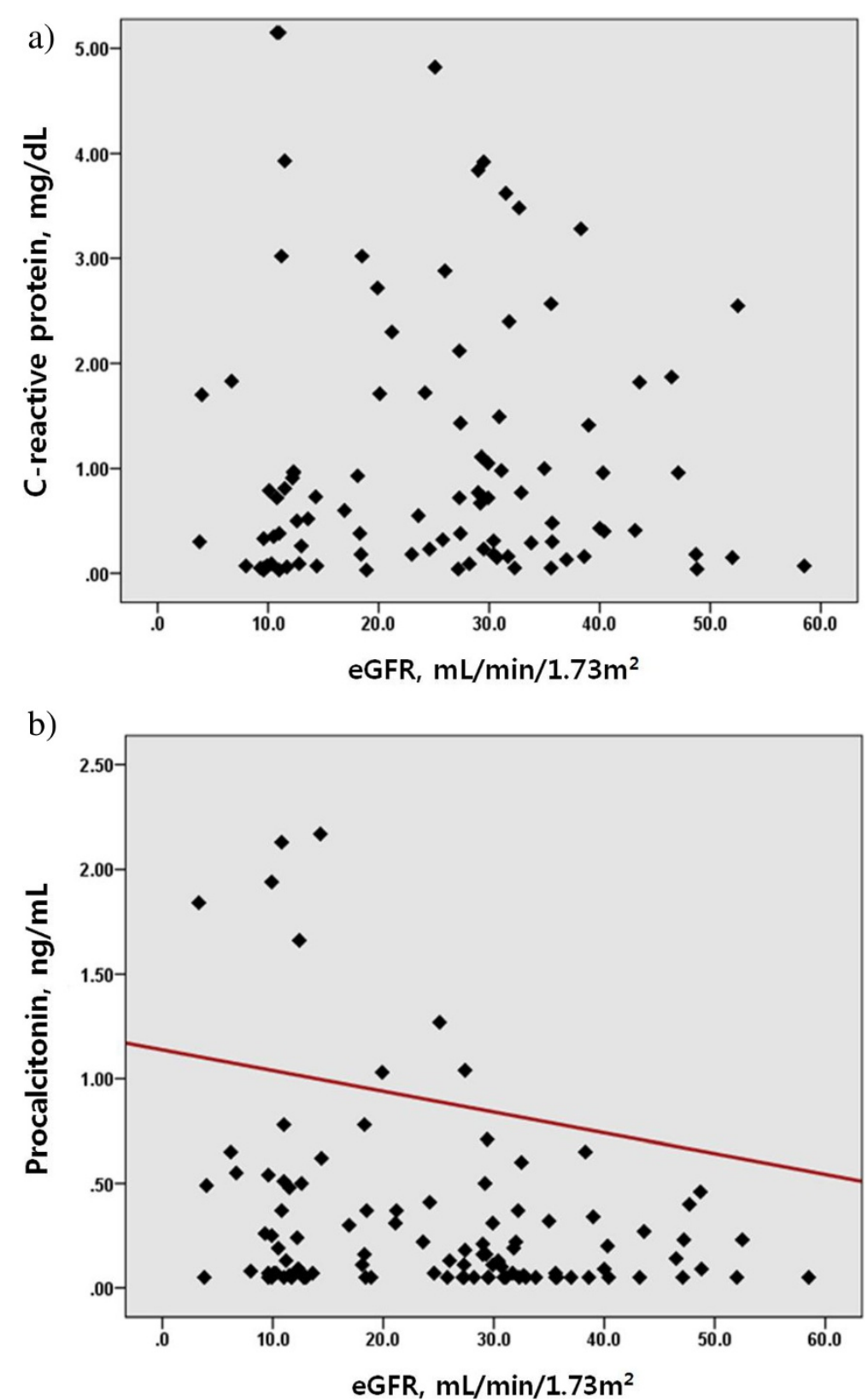

Figure 3 Association between C-reactive protein (CRP) and procalcitonin (PCT) levels and estimated glomerular filtration rate (eGFR) in patients with impaired renal function. Spearman correlation analysis was used to obtain $r$ - and $P$-values. (a) CRP was not correlated with eGFR ( $r=0.037, P=0.70)$. (b) PCT was significantly correlated with eGFR $(r=0.247, P=0.01)$. 


\section{Abbreviations}

AUC: areas under the respective curve; CKD: chronic kidney disease; CRP: C-reactive protein; DM: diabetes mellitus; eGFR: estimated glomerular filtration rate; HD: hemodialysis; HTN: hypertension; IQR: interquartile range; MDRD: modification of diet in renal disease; PCT: procalcitonin; PD: peritoneal dialysis; ROC: receiver operating characteristic; RRT: renal replacement therapy; SIRS: systemic inflammatory response syndrome.

\section{Competing interests}

The authors declare that they have no competing interests.

\section{Authors contributions}

JHP analyzed data, interpreted results, prepared figures, and drafted and revised the manuscript. DHK analyzed data. HRJ designed this research, interpreted results, prepared figures, and revised the manuscript. MJK assisted in the statistical methodology and interpreted results. SHJ assisted in the statistical methodology and approved the manuscript. JEL participated in the design of the study and approved the manuscript. WH participated in the design of the study and approved the manuscript. DJK participated in the design of the study and approved the manuscript. YGK participated in the design of the study and approved the manuscript. HYO participated in the design of the study and approved the manuscript. All authors read and approved the final manuscript.

\section{Acknowledgements}

We thank all faculties of the division of infectious disease and the critical care team in Samsung Medical Center, Sungkyunkwan University School of Medicine for their devotional effort in the field of clinical practice and helpful advice.

\section{Author details}

'Division of Nephrology, Department of Medicine, Samsung Medical Center, Sungkyunkwan University School of Medicine, 81 Irwon-ro, Gangnam-gu, Seoul 135-710, Republic of Korea. ${ }^{2}$ Biostatistics and clinical epidemiology center, Samsung Medical Center, Sungkyunkwan University School of Medicine, Seoul, Korea.

Received: 11 May 2014 Accepted: 4 November 2014

Published online: 19 November 2014

\section{References}

1. James MT, Quan H, Tonelli M, Manns BJ, Faris P, Laupland KB, Hemmelgarn $\mathrm{BR}: \mathrm{CKD}$ and risk of hospitalization and death with pneumonia. Am J Kidney Dis 2009, 54:24 32.

2. Dalrymple LS, Katz R, Kestenbaum B, de Boer IH, Fried L, Sarnak MJ, Shlipak MG: The risk of infection-related hospitalization with decreased kidney function. Am J Kidney Dis 2012, 59:356 363.

3. Girndt M, Sester M, Sester U, Kaul H, Kohler H: Molecular aspects of T- and B-cell function in uremia. Kidney Int Supp/ 2001, 78:S206 S211.

4. Herbelin A, Nguyen AT, Zingraff J, Urena P, Descamps-Latscha B: Influence of uremia and hemodialysis on circulating interleukin-1 and tumor necrosis factor alpha. Kidney Int 1990, 37:116 125

5. Panichi V, Migliori M, De Pietro S, Taccola D, Bianchi AM, Norpoth M, Metelli MR, Giovannini L, Tetta C, Palla $R$ : $C$ reactive protein in patients with chronic renal diseases. Ren Fail 2001, 23:551 562

6. Pepys MB, Hirschfield GM: C-reactive protein: a critical update. J Clin Invest 2003, 111:1805 1812.

7. Herget-Rosenthal S, Marggraf G, Pietruck F, Husing J, Strupat M, Philipp T, Kribben A: Procalcitonin for accurate detection of infection in haemodialysis. Nephrol Dial Transplant 2001, 16:975 979.

8. Castelli GP, Pognani C, Meisner M, Stuani A, Bellomi D, Sgarbi L: Procalcitonin and C-reactive protein during systemic inflammatory response syndrome, sepsis and organ dysfunction. Crit Care 2004, 8:R234 R242.

9. Simon L, Gauvin F, Amre DK, Saint-Louis P, Lacroix J: Serum procalcitonin and C-reactive protein levels as markers of bacterial infection: a systematic review and meta-analysis. Clin Infect Dis 2004, 39:206 217.

10. Lu XL, Xiao ZH, Yang MY, Zhu YM: Diagnostic value of serum procalcitonin in patients with chronic renal insufficiency: a systematic review and meta-analysis. Nephrol Dial Transplant 2013, 28:122 129.
11. Tang BMP, Eslick GD, Craig JC, McLean AS: Accuracy of procalcitonin for sepsis diagnosis in critically ill patients: systematic review and metaanalysis. Lancet Infect Dis 2007, 7:210 217.

12. Piraino B, Bailie GR, Bernardini J, Boeschoten E, Gupta A, Holmes C, Kuijper J, Li PK-T, Lye W-C, Mujais S: ISPD guidelines/recommendations. Perit Dial Int 2005, 25:107 131.

13. American College of Chest Physicians/Society of Critical Care Medicine Consensus Conference: definitions for sepsis and organ failure and guidelines for the use of innovative therapies in sepsis. Crit Care Med 1992, 20:864 874

14. DeLong ER, DeLong DM, Clarke-Pearson DL: Comparing the areas under two or more correlated receiver operating characteristic curves: a nonparametric approach. Biometrics 1988, 44:837 845.

15. Dumea R, Siriopol D, Hogas S, Mititiuc I, Covic A: Procalcitonin: diagnostic value in systemic infections in chronic kidney disease or renal transplant patients. Int Urol Nephrol 2014, 46:461 468.

16. Jacobs JW, Lund PK, Potts JT Jr, Bell NH, Habener JF: Procalcitonin is a glycoprotein. J Biol Chem 1981, 256:2803 2807.

17. Herget-Rosenthal S, Klein T, Marggraf G, Hirsch T, Jakob HG, Philipp T, Kribben A: Modulation and source of procalcitonin in reduced renal function and renal replacement therapy. Scand J Immunol 2005, 61:180 186.

18. Dahaba AA, Rehak PH, List WF: Procalcitonin and C-reactive protein plasma concentrations in nonseptic uremic patients undergoing hemodialysis. Intensive Care Med 2003, 29:579 583.

19. Schmidt M, Burchardi C, Sitter T, Held E, Schiffl H: Procalcitonin in patients undergoing chronic hemodialysis. Nephron 2000, 84:187 188.

20. Meisner M, Schmidt J, Huttner H, Tschaikowsky K: The natural elimination rate of procalcitonin in patients with normal and impaired renal function. Intensive Care Med 2000, 26:S212 S216.

21. Sitter T, Schmidt M, Schneider S, Schiffl H: Differential diagnosis of bacterial infection and inflammatory response in kidney diseases using procalcitonin. J Nephrol 2002, 15:297 301.

22. Opatrna S, Klaboch J, Opatrny K Jr, Holubec L, Tomsu M, Sefrna F, Topolcan O: Procalcitonin levels in peritoneal dialysis patients. Perit Dial Int 2005, 25:470 472.

23. Claeys R, Vinken S, Spapen H, ver Elst K, Decochez K, Huyghens L, Gorus FK: Plasma procalcitonin and C-reactive protein in acute septic shock: clinical and biological correlates. Crit Care Med 2002, 30:757 762.

24. Meisner M, Tschaikowsky K, Palmaers T, Schmidt J: Comparison of procalcitonin (PCT) and C-reactive protein (CRP) plasma concentrations at different SOFA scores during the course of sepsis and MODS. Crit Care 1999, 3:45 50

25. Karzai W, Oberhoffer M, Meier-Hellmann A, Reinhart K: Procalcitonin a new indicator of the systemic response to severe infections. Infection 1997, 25:329 334

26. Oberhoffer M, Stonans I, Russwurm S, Stonane E, Vogelsang H, Junker U, Jager L, Reinhart K: Procalcitonin expression in human peripheral blood mononuclear cells and its modulation by lipopolysaccharides and sepsis-related cytokines in vitro. J Lab Clin Med 1999, 134:49 55.

\section{doi:10.1186/s13054-014-0640-8}

Cite this article as: Park et al:: Clinical relevance of procalcitonin and C-reactive protein as infection markers in renal impairment: a cross-sectional study. Critical Care 2014 18:640.

\section{Submit your next manuscript to BioMed Central and take full advantage of:}

$\otimes$ Convenient online submission

$\otimes$ Thorough peer review

$\otimes$ No space constraints or color $\bowtie$ gure charges

$\bigotimes$ Immediate publication on acceptance

Q Inclusion in PubMed, CAS, Scopus and Google Scholar

$\otimes$ Research which is freely available for redistribution 